Maarten Uijt de Haag, Svenja Huschbeck, Joel Huff

\title{
sUAS Swarm Navigation using Inertial, Range Radios and Partial GNSS
}

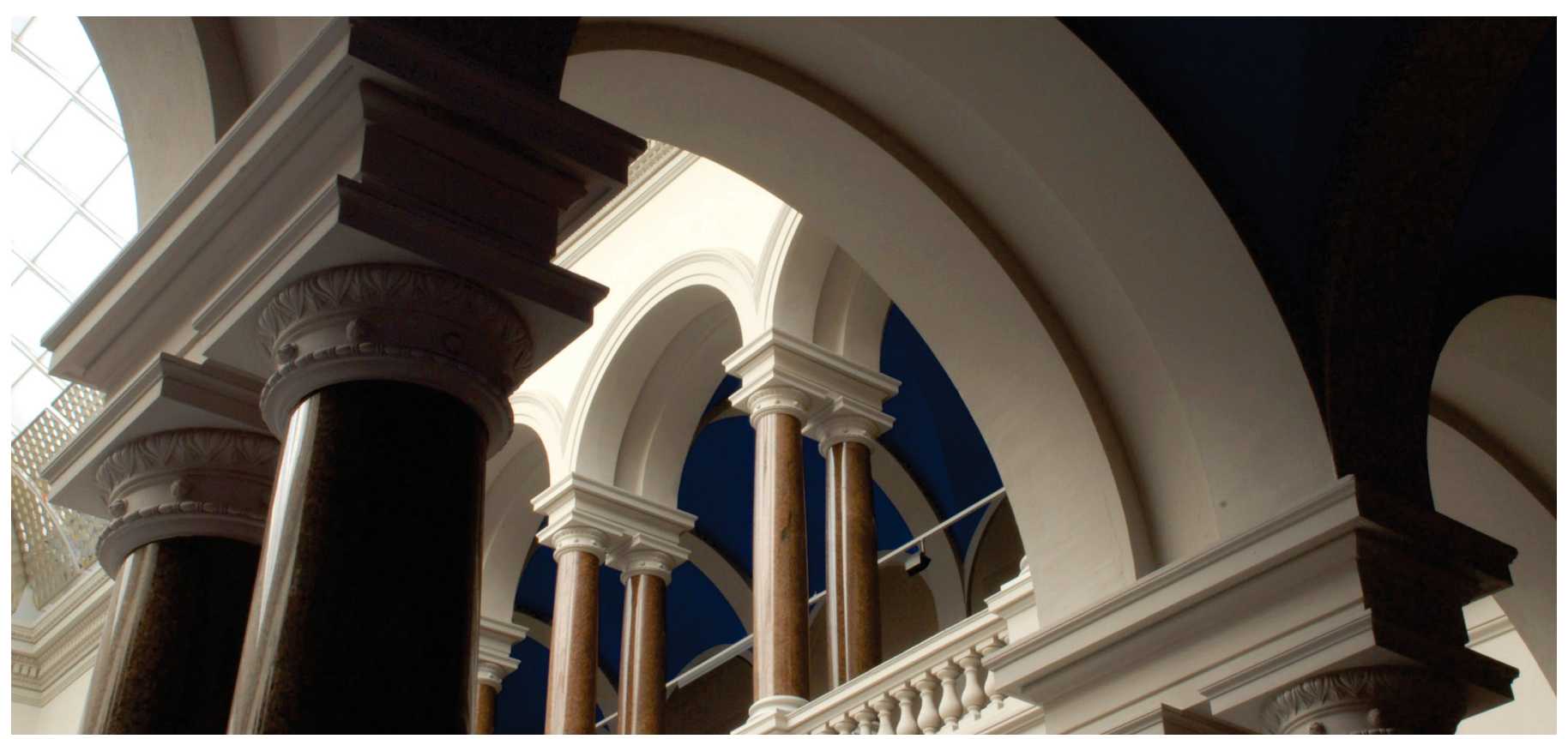

Uijt de Haag, Maarten; Huschbeck, Svenja; Huff, Joel (2019): sUAS Swarm Navigation using Inertial, Range Radios and Partial GNSS. Presented at 2019 IEEE/AIAA 38th Digital Avionics Systems Conference (DASC). 


\title{
sUAS Swarm Navigation using Inertial, Range Radios and Partial GNSS
}

\author{
Maarten Uijt de $\mathrm{Haag}^{1}$ and Svenja Huschbeck², TU Berlin, Germany \\ Joel Huff ${ }^{3}$, Ohio University, Ohio, U.S.A
}

This paper was first published in the proceedings of the DASC 2019 in San Diego and does not show any changes in content to the primary publication. The full reference to the first publication is:

Maarten Uijt de Haag, Svenja Huschbeck, Joel Huff (2019). sUAS Swarm Navigation using Inertial, Range Radios and Partial GNSS. DASC 2019.

\begin{abstract}
Small Unmanned Aerial Systems (sUAS) operations are increasing in demand and complexity. Using multiple cooperative sUAS (i.e. a swarm) can be beneficial and is sometimes necessary to perform certain tasks (e.g., precision agriculture, mapping, surveillance) either independent or collaboratively. However, controlling the flight of multiple sUAS autonomously and in real-time in a challenging environment in terms of obstacles and navigation requires highly accurate absolute and relative position and velocity information for all platforms in the swarm. This information is also necessary to effectively and efficiently resolve possible collision encounters between the sUAS. In our swarm, each platform is equipped with a Global Navigation Satellite System (GNSS) sensor, an inertial measurement unit (IMU), a baro-altimeter and a relative range sensor (range radio). When GNSS is available, its measurements are tightly integrated with IMU, baro-altimeter and range-radio measurements to obtain the platform's absolute and relative position. When GNSS is not available due to external factors (e.g., obstructions, interference), the position and velocity estimators switch to an integrated solution based on IMU, baro and relative range measurements. This solution enables the system to maintain an accurate relative position estimate, and reduce the drift in the swarm's absolute position estimate as is typical of an IMU-based system.

Multiple multi-copter data collection platforms have been developed and equipped with GNSS, inertial sensors and range radios, which were developed at Ohio University. This paper outlines the underlying methodology, the platform hardware components (three multi-copters and one ground station) and analyzes and discusses the performance using both simulation and sUAS flight test data.
\end{abstract}

\section{KEYWORDS}

component; aircraft state awareness; energy awareness; predictive alerting; sensor integration

\footnotetext{
${ }^{1}$ Professor of Chair, Chair of Flight Guidance \& Air Transport, TU Berlin

2 Research Assistant and PhD candidate, Chair of Flight Guidance \& Air Transport, TU Berlin

${ }^{3}$ Graduate Research Assistant, Department of Electrical Engineering and Computer Science, Ohio University
} 


\section{INTRODUCTION}

As modern small unmanned aircraft systems (sUAS) grow more mature and the sUAS hardware comes down in price, sUAS are being applied to more and more problems. For example, an sUAS can be used as a highly mobile camera or a sensor set, capable of performing remote mapping or other sensing missions. With just a sensor payload, sUAS have been studied and tested for 3D mapping [1], forest inventory support [2], surveillance [3], and many more applications. These applications all require a high degree of accuracy for the absolute position of the aircraft to relate the sensor information to one or more points on Earth.

Though most studies are currently being performed on single sUAS, many applications may benefit in some way from multiple, cooperative sUAS. In the context of this paper, this network of two or more cooperative sUAS is referred to as an sUAS swarm. 3D mapping methods, like the monocular camera methods discussed in [1], could benefit from having multiple sUAS in the same area, finishing the mapping job quicker and more thoroughly. With monocular camera mapping, it is important to have as many angles as possible of the same object, while still being able to relate the images to a common reference frame. This is typically accomplished by a single sUAS moving around the object, but this task could also be performed cooperatively with multiple sUAS. The forest inventory method discussed in [2] could also benefit from utilizing a swarm of sUAS, for similar reasons. In addition to getting the job done faster, it may also be possible for a swarm of cooperative sUAS to aid each other in navigation of a GNSS-denied under-the-canopy environment. If the sUAS were using a Simultaneous Localization and Mapping (SLAM) method such as implemented in [4], they would also be able to exchange landmark information, allowing loop closure and better map building across the swarm.

The authors of [3] discuss a cooperative surveillance system, in which multiple sUAS observe and track multiple targets, showing that surveillance can benefit from sUAS cooperation. This cooperation ensures that the sUAS are not just blindly tracking a single target; the sUAS can make intelligent decisions so that members don't track the same target, members can refuel during optimal windows, and the surveyed area is properly covered, while the information gained by the swarm is maximized.

To make these multi-platform applications possible, many researchers have begun to address the navigation challenges of sUAS swarms. During formation flight, sUAS swarm navigation can be broken down into two distinct problems: absolute (global) positioning of the individual members,

and the position of the members of the swarm relative to the other members. The latter relative navigation solution can be defined by separation vectors between each of the members. 


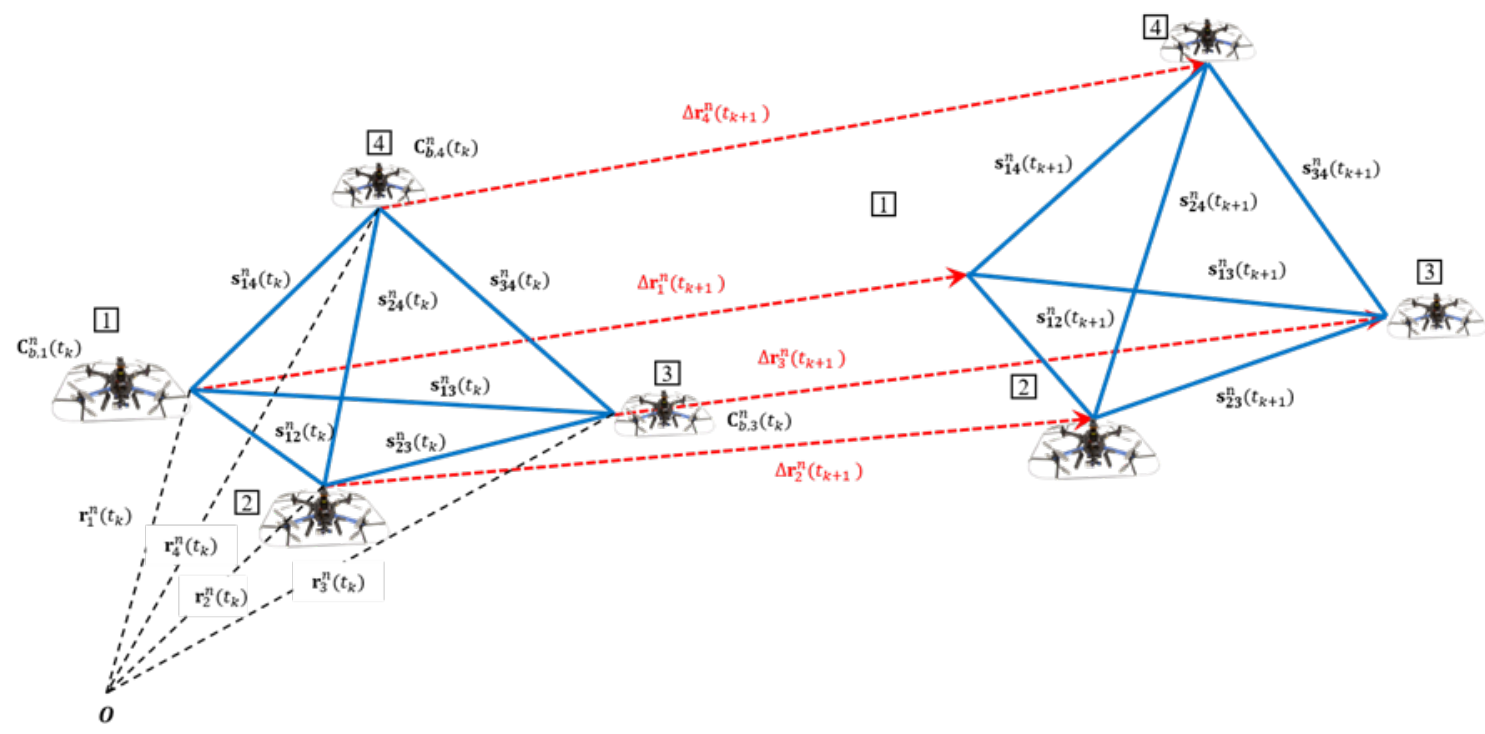

Figure 1. Swarm geometry of four sUAS as a function of time.

In the example shown in Figure 1, each individual member must calculate its absolute position vector $\boldsymbol{r}_{i}^{n}$ while also assuring that the separation vectors $\boldsymbol{s}_{i k}^{n}$ between it and every other member are as accurate as possible. Here ' $n$ ' refers to the navigation frame in which all absolute coordinates and vectors are expressed (e.g. lat-lon-height, Earth Centered Earth Fixed or East North Up). Note that this coordinate frame must be the same for all sUAS. In many scenarios these separation vectors may even be more important than the global positions of the members, to avoid collisions or perform complex cooperative tasks.

Relative GNSS-based navigation methods, such as the one presented in previous work by the authors [5] work well for this task, given that all members have GNSS capability. Because many errors are the same for receivers in close proximity, the separation vectors can be easily calculated and remain accurate at the decimeter level. However, in the event of an GNSS outage or a compromised or malfunctioning receiver, the ability to calculate accurate separation vectors significantly degrades. This is a big problem for operation of cooperative swarms in GNSS-denied or semi-denied environments: urban canyon environments for bridge or construction inspection, under-the-canopy operation during environmental monitoring or inventory tasks, city traffic surveillance, etc. If a swarm seeks to function in such environments, members must be able to perform relative navigation when some or all members are GNSS-denied.

UAS mounted ultra-wideband (UWB) range radios are a perfect fit to aid in the relative navigation task as these sensors provide the ranges (i.e. $\rho_{i k}=\left\|\boldsymbol{s}_{i k}\right\|$ ) between two sUAS in the swarm. Although they do not provide the actual separation vector, multi-lateration among the sensed ranges can be used to estimate the relative separation vectors in a local frame rigidly attached to the swarm. When combined with sensors that offer an absolute positioning or orientation capability (i.e., with respect to a global navigation reference frame), these relative separation vectors can be translated and rotated to a global frame. The implications of the Inertial Navigation System (INS) and UWB sensor fusion approach have been discussed in [7] where it is shown that INS/UWB 
integrated is reasonably accurate in practice. The methodology behind the results in that paperan extended Kalman filter - is computationally efficient, and therefore perfect for sUAS with limited computational resources. The author went on to apply this method to an indoor positioning system with great success [8].

Note that within this paper a centralized approach is discussed that assumes that the range measurements, baro-altimeter measurements and inertial measurements of each sUAS is transmitted to a central location (e.g., one or all of the sUAS or a ground station) and then used for absolute and relative navigation.

\section{MULTI-LATERATION AMBIGUITIES}

The integration method proposed in the next section exploits the fact that the range radios provide range constraints between the various members of the sUAS swarm. It is important to realize that these constraints fix the relative position of the sUAS within the swarm but do not constraint the swarm's orientation and location in 3D space as is illustrated in Figure 2.

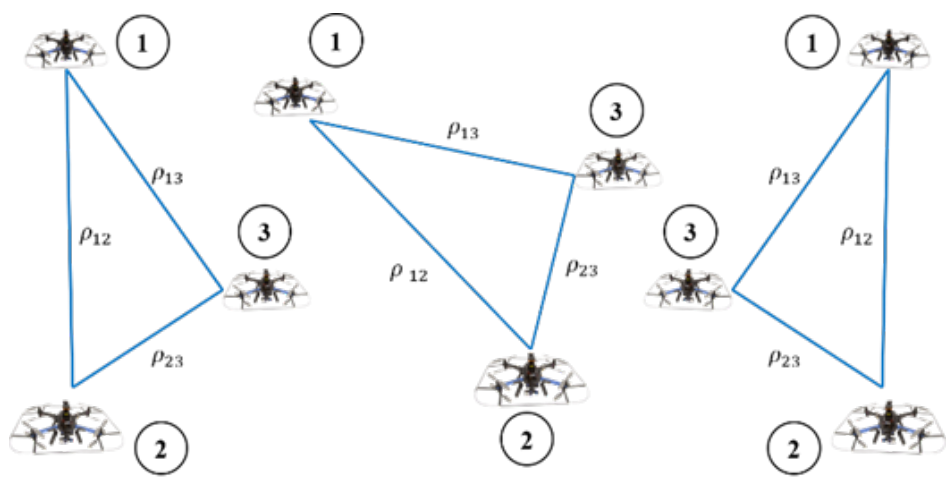

Figure 2. Absolute orientation and position under range constraints (2D example).

When one member of the swarm, has an absolute position capability, the swarm is anchored to this location with associated position uncertainty, but can still rotate freely about this point in 3D. When two of the members ( 1 and 2 ) have knowledge of their global position estimate, but member 3 does not, the ambiguity is reduced to 1-degree of freedom as shown in Figure 3.

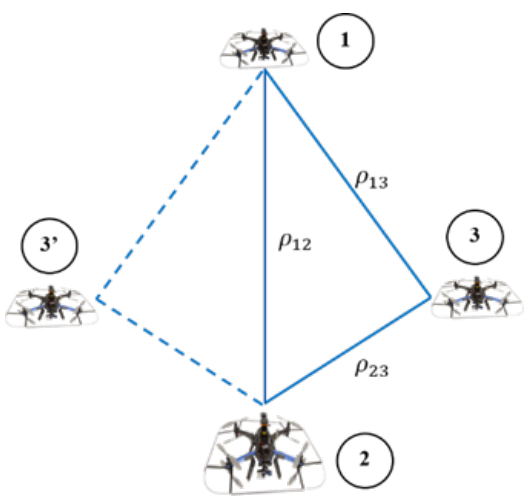

Figure 3. Ambiguity example with two sUAS tight to a global position (2D example). 
With known ranges $\rho_{12}, \rho_{13}$, and $\rho_{23}$, member 3 's position is ambiguous: it could either be in at position 3 or position 3' in 2D. In three dimensions (3D) the location of sUAS 3 could be on a circle. In 3D the ambiguity could again be reduced to two points when the altitude is known from a baroaltimeter, for example, as illustrated in Figure 4.

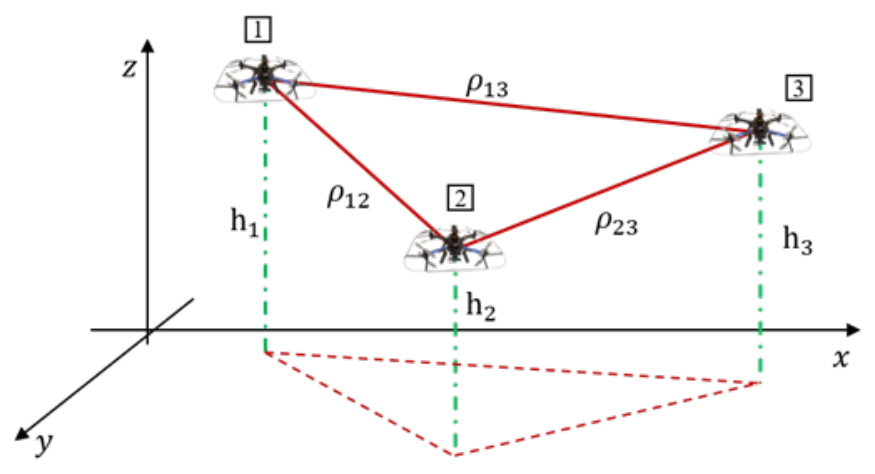

Figure 4. Observability check implementation.

\section{INTEGRATION APPROACH}

\subsection{Primary Mechanization}

Multiple filter mechanizations exist that could potentially integrate range measurements and baroaltimeter measurements with inertial and partial GNSS measurements to obtain absolute and relative navigation solutions for the members of a swarm of sUAS either centralized and decentralized. In this paper we use a centralized complementary extended Kalman filter (CEKF). However, alternate filters such as particle filters (PF) or graph-based methods could be used. These methods will be the focus of a later paper. The basic equations for the CEKF used here are provided in Equations (1) through (7) and can be found in many textbooks such as [9].

System: dynamics model

$$
\mathbf{x}\left(t_{k}\right)=\mathbf{g}\left[\mathbf{x}\left(t_{k-1}\right)\right]+\mathbf{w}\left(t_{k}\right)
$$

System: measurement model

$$
\mathbf{z}\left(t_{k}\right)=\mathbf{h}\left[\mathbf{x}\left(t_{k}\right)\right]+\mathbf{v}\left(t_{k}\right)
$$

Kalman filter: update step

$$
\begin{gathered}
\hat{\mathbf{x}}\left(t_{k}\right)=\hat{\mathbf{x}}^{-}\left(t_{k}\right)+\mathbf{K}_{k}\left[\mathbf{z}_{k}\left(t_{k}\right)-\mathbf{h}\left[\hat{\mathbf{x}}^{-}\left(t_{k}\right)\right]\right] \\
\mathbf{K}\left(t_{k}\right)=\frac{\mathbf{P}^{-}\left(t_{k}\right) \mathbf{H}^{T}}{\left[\mathbf{H}\left(t_{k}\right) \mathbf{P}^{-}\left(t_{k}\right) \mathbf{H}^{T}\left(t_{k}\right)+\mathbf{R}\right]} \\
\mathbf{P}\left(t_{k}\right)=\left[\mathbf{I}-\mathbf{K}\left(t_{k}\right) \mathbf{H}\left(t_{k}\right)\right] \mathbf{P}^{-}\left(t_{k}\right)
\end{gathered}
$$

Kalman filter: prediction step

$$
\hat{\mathbf{x}}^{-}\left(t_{k}\right)=\hat{\mathbf{x}}\left(t_{k}\right)+\int_{t_{k-1}}^{t_{k}} \mathbf{g}(\hat{\mathbf{x}}, t) d t
$$




$$
\begin{gathered}
\mathbf{P}^{-}\left(t_{k}\right)=\boldsymbol{\Phi}\left(t_{k-1}\right) \mathbf{P}\left(t_{k-1}\right) \boldsymbol{\Phi}^{T}\left(t_{k-1}\right) \\
+\int_{t_{k-1}}^{t_{k}} \boldsymbol{\Phi}\left(t_{k-1} \mid t\right) \mathbf{Q}\left(t_{k-1}\right) \boldsymbol{\Phi}^{T}\left(t_{k-1} \mid t\right) d t
\end{gathered}
$$

Where superscript '-' indicates that the quantity is a prediction. The 15-dimensional error state for UAS ' $i$ ' is given by:

$$
\mathbf{x}_{i}=\left[\begin{array}{lllll}
\delta \mathbf{r}_{i} & \delta \mathbf{v}_{i} & \delta \boldsymbol{\Psi}_{\boldsymbol{i}} & \delta \mathbf{a}_{i}^{b} & \delta \boldsymbol{\omega}_{i}^{b}
\end{array}\right]^{T}
$$

where $\delta r_{i}$ is the 3D position error of the inertial on SUAS $i, \delta \mathbf{v}_{\mathrm{i}}$ is the $3 \mathrm{D}$ velocity error, $\delta \psi_{\mathrm{i}}$ is the attitude error, $\delta \boldsymbol{a}_{i}^{b}$ bis the 3D accelerometer bias, and $\delta \omega_{i}^{b}$ is the 3D gyro bias. The state transition matrix is the short-term IMU error state transition matrix such as the ones defined found in [10] and [11]. This will allow the inertial solution to run freely between filter updates. After each update step, the inertial solution will be corrected by the error estimates. For all ' $N$ ' INS systems in the swarm, the state vector is updated to a new $15 N \times 1$ state vector which includes the errors of all INSs:

$$
\mathbf{x}=\left[\begin{array}{llll}
\mathbf{x}_{1} & \mathbf{x}_{2} & \cdots & \mathbf{x}_{N}
\end{array}\right]
$$

The range between any two sUAS can be computed using their respective INS position estimates, and expressed in terms of the true relative position and an additional error term that combines the INS errors from ' $i$ ' and ' $k$ ', or:

$$
\begin{gathered}
\rho_{i k_{\text {ins }}}=\left\|\tilde{\mathbf{r}}_{i, i n s}-\tilde{\mathbf{r}}_{k, \text { ins }}\right\| \\
=\left\|\mathbf{r}_{i, t r u e}+\delta \mathbf{r}_{i, \text { ins }}-\mathbf{r}_{j, \text { true }}-\delta \mathbf{r}_{k, \text { ins }}\right\| \\
=\left\|\Delta \mathbf{r}_{i k, \text { true }}-\delta \mathbf{r}_{i, \text { ins }}\right\|=\left\|\mathbf{s}_{i k}-\delta \mathbf{r}_{i k, \text { ins }}\right\|
\end{gathered}
$$

where $\mathbf{r}_{\mathrm{i}, \text { true }}$ is the true position of UAS $i, \delta \boldsymbol{r}_{i, i n s}$ is the position error of the INS of UAS $i, \delta \mathbf{r}_{k, \text { ins }}$ is the position error of the INS of UAS $k$. Equation (12) can be linearized with respect to the involved sUAS INS position estimates.

$$
\begin{gathered}
\tilde{\rho}_{i k_{i n s}} \approx \rho_{i k_{\text {true }}}+\frac{\mathbf{s}_{i k}^{T}}{\rho_{i k}} \delta \mathbf{r}_{i k, i n s} \\
=\rho_{i k_{\text {true }}}+\underbrace{\mathbf{u}_{i k}^{T} \delta \mathbf{r}_{i k i n s}}_{\delta \rho_{i k_{i n s}}}
\end{gathered}
$$

The measurement vector is derived from available on range radio measurements, baroaltimeter measurements and inertial position estimates:

$$
\mathbf{z}=\left[\begin{array}{c}
z_{12} \\
z_{13} \\
\vdots \\
z_{i j} \\
\vdots \\
z_{(N-1) N} \\
z_{\text {baro,1 }} \\
\vdots \\
z_{\text {baro,N }}
\end{array}\right]
$$


The row elements of Equation (15) are given by the various available range differences in the swarm:

$$
\begin{gathered}
z_{i k}=\tilde{\rho}_{i k_{i n s}}-\tilde{\rho}_{i k_{r r}} \\
=\rho_{i k_{t r u e}}+\delta \rho_{i k_{i n s}}-\rho_{i k_{t r u e}}+v_{r r} \\
=\delta \rho_{i k_{i n s}}+v_{r r}
\end{gathered}
$$

where $\delta \rho_{i k_{\text {ins }}}$ is the error due to the contributions of two INSs involved, and $v_{r r}$ is the range noise error of the range radio. Equation (15) can be expressed in the inertial position error terms as follows:

$$
z_{i k}=\delta \rho_{i k_{i n s}}+v_{r r} \approx-\mathbf{u}_{i k}^{T} \delta \mathbf{r}_{i k, i n s}+v_{r r}
$$

The baro-altimeter measurement is much simpler, as it is already linear:

$$
\begin{gathered}
z_{\text {baro }, j}=h_{j_{\text {ins }}}-h_{j_{\text {baro }}} \\
=\left(h_{j_{\text {true }}}+\delta r_{j_{z, \text { ins }}}\right)-\left(h_{j_{\text {true }}}+v_{\text {baro }}\right) \\
=\delta r_{j_{z, \text { ins }}}+v_{\text {baro }}
\end{gathered}
$$

where $\delta r_{j_{z, \text { ins }}}$ is the $\mathrm{z}$-component of the position vector computed by the INS. The results of equations (16) and (17) can be used to setup the H-matrix.

\subsection{Observability}

In addition to the sensor information used in the above model, support was added for feeding in a specific member's GNSS-derived position, as well as magnetometer readings. This extra sensor information modifies the measurement and observation matrices in a manner similar to the addition of baro-altimeter readings in Equation (17). This allows the testing of a 6-member swarm where arbitrarily chosen members have GNSS position estimates available and others have not. This loosely-coupled approach for the GNSS readings was chosen to simplify the filter states and processing time, though a tightly-coupled approach is certainly possible and will be addressed in future work.

Additionally, an observability analysis was added by analyzing the rank of the observability matrix (shown below) is calculated [12]:

$$
\mathcal{O}=\left[\begin{array}{c}
\boldsymbol{H} \\
\boldsymbol{H} \boldsymbol{\phi} \\
\boldsymbol{H} \Phi^{2} \\
\vdots \\
\boldsymbol{H} \boldsymbol{\Phi}^{k-1}
\end{array}\right]
$$

where $k$ is the number of states in the system. The filter is said to be fully observable if the rank of $\boldsymbol{O}$ is greater than $15 n$, the number of states in the filter. From the system model, the yaw component of the angle error state is colinear with gravity, and therefore never fully observable. With this in mind, if the rank of $\boldsymbol{O}$ is at least $14 n$, the error states should be observable. However, due to the heavy processing time related to building and evaluating the rank of $\boldsymbol{O}$, this observability check only 
occurs at initialization and if the sensor configuration has changed (i.e. a member enters or leaves a GNSS-denied area).

\section{TEST SETUP AND METRICS}

\subsection{Test Setup and Trajectories}

To evaluate the proposed method using real sUAS platforms, a data collection was performed using three manually-flown custom-built platforms shown in Figure 5. All three platforms are based on 3DR X8 frames, controlled by a PixHawk PX4 flight controller running a distribution of Arducopter. Furthermore, each platform is equipped with an Odroid XU4 for onboard processing and data collection, as well as a point-to-multipoint data radio for platform-to-platform and platform-to-ground station communication. Sensor data is collected and logged via the Robotic Operating System (ROS) [10][14]. A laptop ground station controls when data logging is started and stopped via a custom-built Graphical User Interface (GUI).

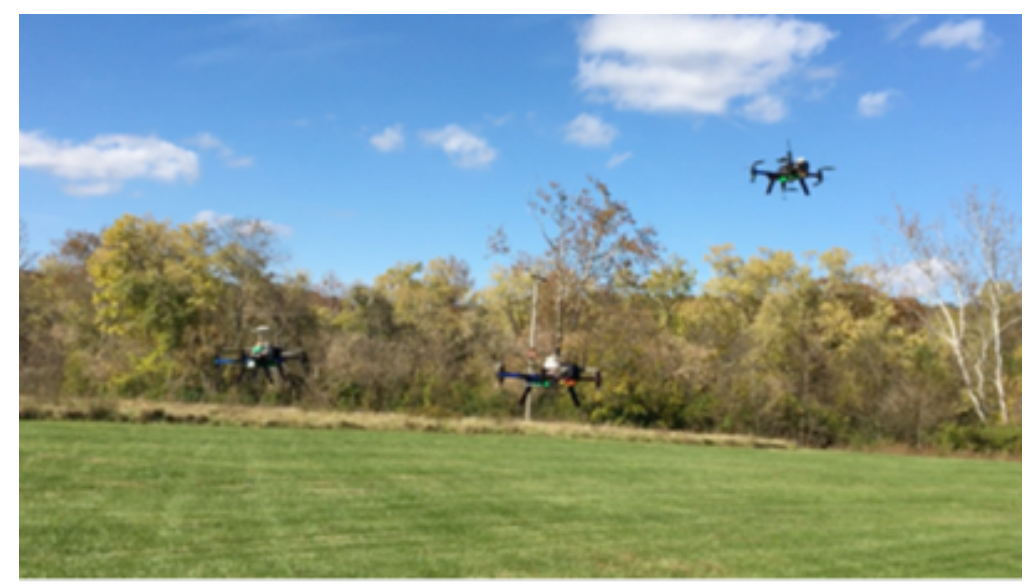

Figure 5. Formation flight at test field at Ohio University.

The platforms were equipped with multiple inertial units of varying costs and qualities, as well as different GNSS receivers: Platform A with a Sensonar STIM300 and a Novatel OEM-615; Platform B with a VectorNav VN-100 and Xsens Mti-1 inertial and a Novatel OEM-615 GNSS receiver, and Platform $\mathbf{C}$ with an Xsens Mti-1 and a U-blox M8T.

During two flights, sensor data was collected and time-tagged. The two flight-data set times were adjusted and then overlaid in post-processing to generate a single data set equivalent to a sixmember flight. The 6 overlaid flight trajectories are shown in Figure 6. 


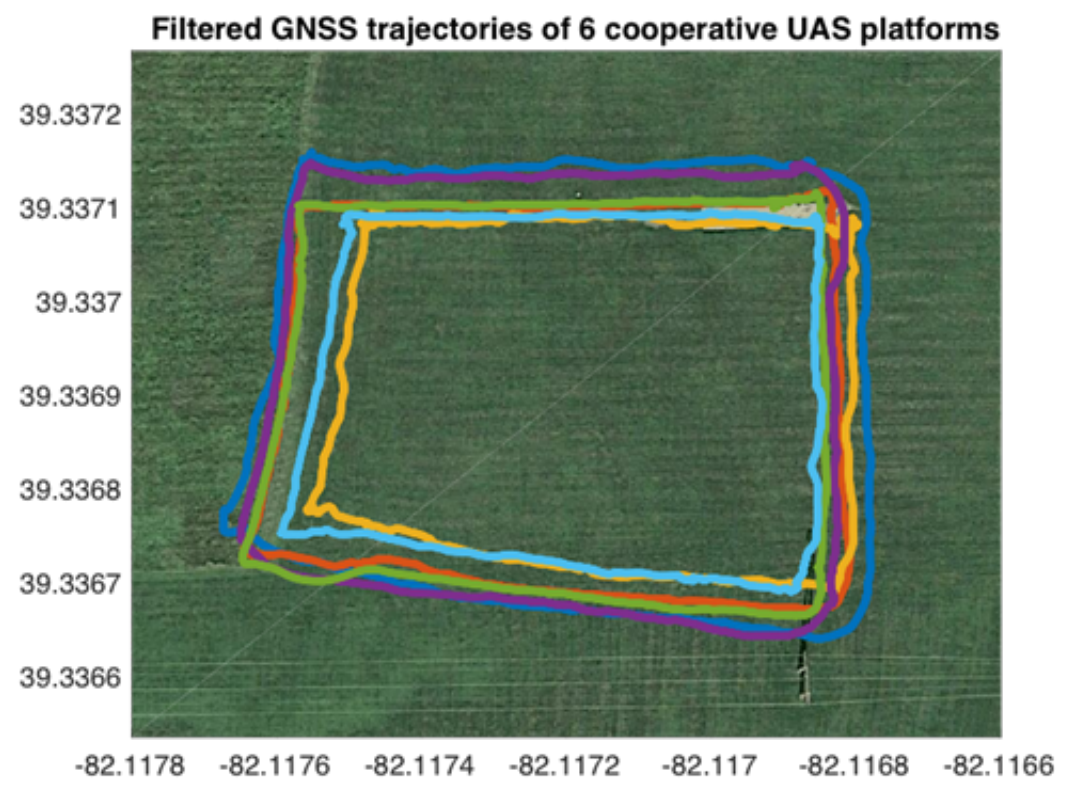

Figure 6. Overlaid flight trajectories.

Range radio measurements were simulated from the GNSS position solutions and noise was added based on the ranging performance of the range radios developed by Ohio University and presented earlier in [5] and [6] and shown in Figure 7.

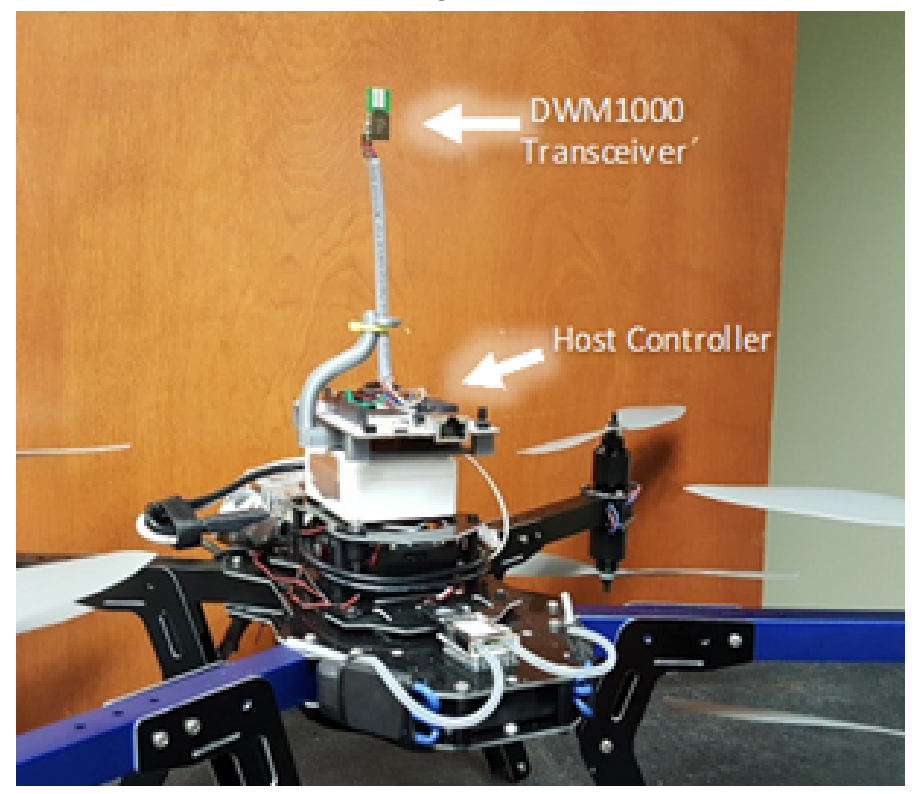

Figure 7. Radio system mounted on X8 octocopter

The performance of this particular radio up to $10 \mathrm{~m}$ is illustrated in Figure 8. 

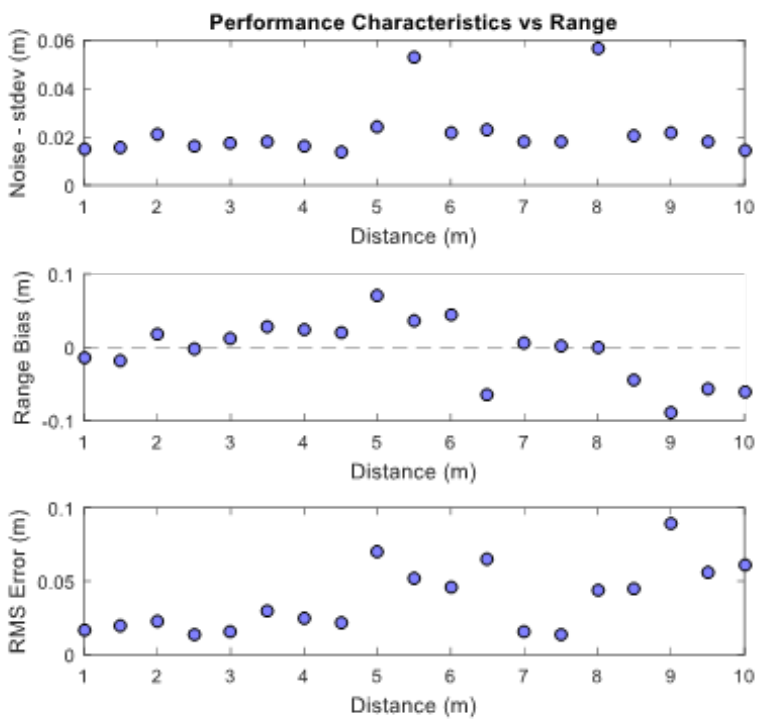

Figure 8. Performance of the RR as a function of distance up to $10 \mathrm{~m}$.

Next, the GNSS data was removed and the proposed filter applied on the remaining data.

\subsection{Evaluation Metrics}

To analyze the effects of the proposed filter, both the absolute and relative position errors have been evaluated. The absolute position error is quantified by the root-mean-square-error (RMSE) between the estimated and true member positions for each dimension. Assessing the relative navigation performance is more complex due to the ambiguities introduced by multi-lateration as discussed in Section 2. To derive this Cartesian position error, a rotation and translation is found that maps the navigation solution as closely as possible to the true trajectory. This is solved by a least-squares estimator called Horn's method [13]. The navigation solution is then transformed by the found rotation and translation and position errors are determined for each member. Additionally, the measurement innovations (the individual range errors) as well as the filter-estimated trajectories have been considered in the following sections.

\section{RESULTS}

\section{$5.1 \quad$ Initial-only Solution}

The inertial-only solution is shown in Figure 9. It is clear that the inertial-only solution is unfit for use; as expected accumulated drift grows to a $7 \mathrm{~km}+$ in just 4 minutes. The members of the swarm drift in entirely different directions, even though they are flying in a loose formation. As a result, one can observed a poor relative navigation solution. 


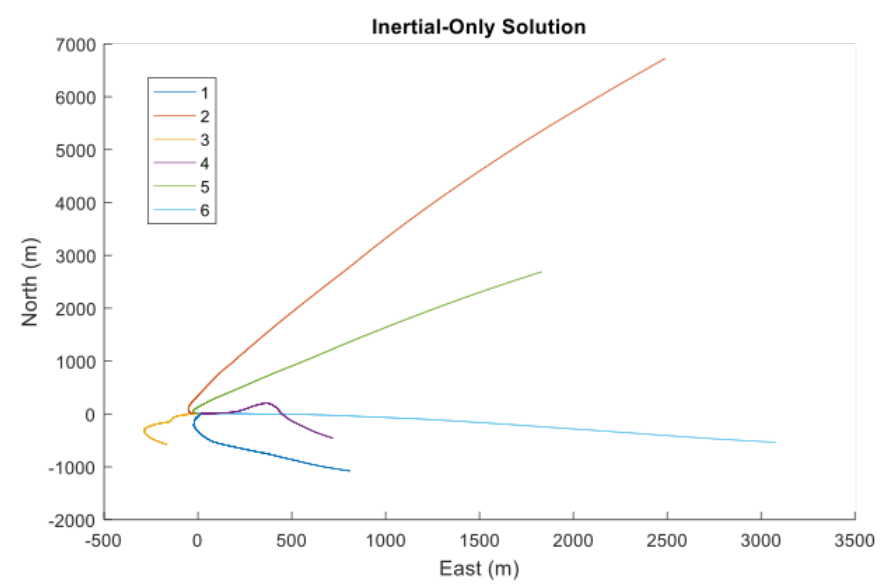

Figure 9. Inertial-only navigation solution.

\subsection{No GNSS-Enabled Members}

The first test did only consider range radio, inertial and barometric altimeter measurements. The results are shown in Figure 10. The plots show a swarm drifting together at a lower drift. Furthermore, a significant performance improvement is achieved in the relative navigation solution. The individual range errors - the errors in the calculated ranges between the filtered and true trajectories - also stay low. This makes sense, as these errors equate to the inputs to the Kalman filter, which attempts to correct them via error estimates.
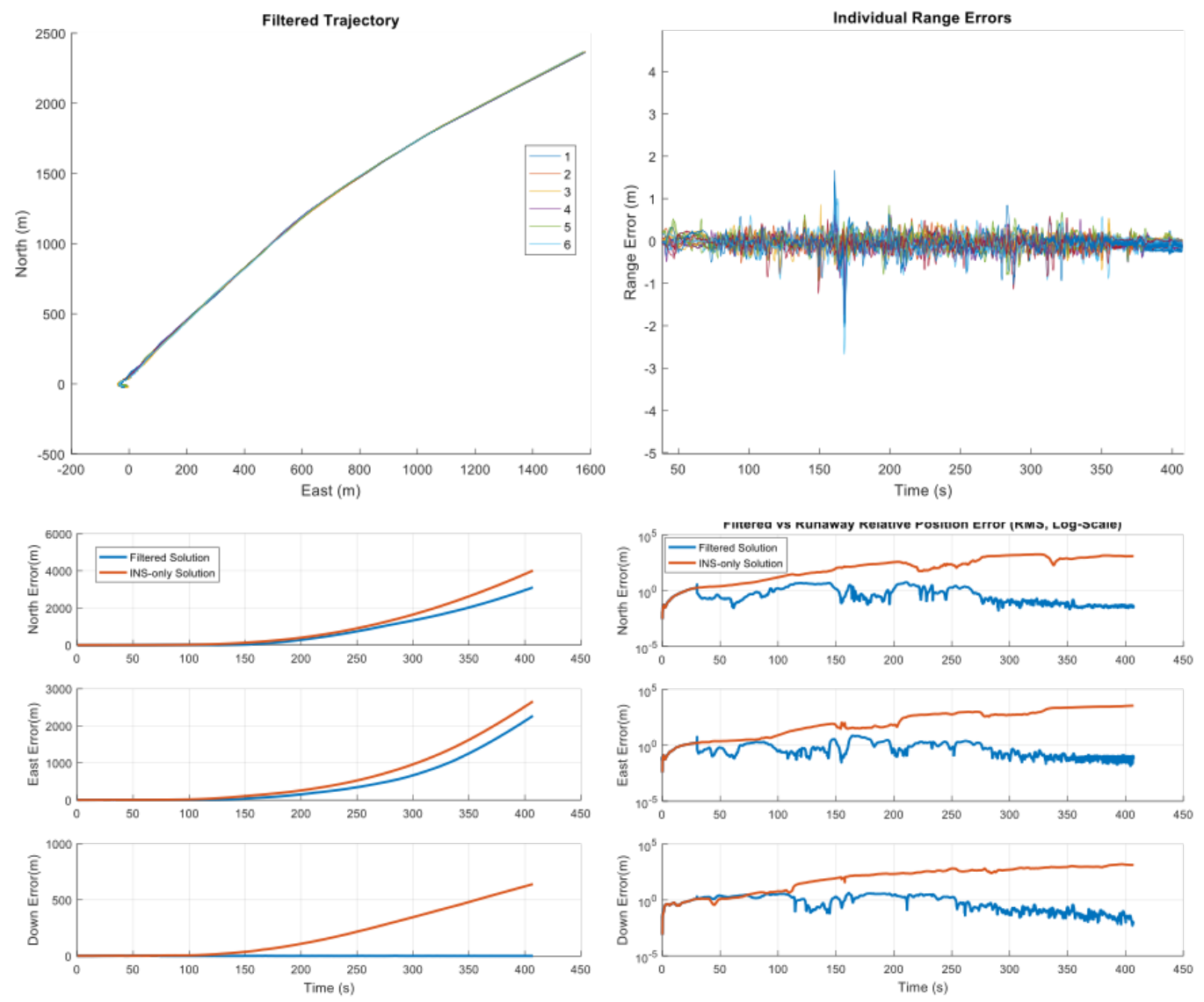

Figure 10. Formation flight at test field at Ohio University. 


\subsection{One GNSS-Enabled Member}

Next, one member of the sUAS swarm is assumed to be resilient to the GNSS interference or other denial-of-service and has, therefore, a valid GNSS solution in addition to the basic sensor configuration (i.e. INS and barometric altimeters). This causes the swarm to be anchored to an absolute position, as the single GNSS solution will constraint the rest of the members. The results of this configuration are shown in Figure 11. With a single GNSS-aided member in the swarm (Octocopter 2 ), every member's solution becomes more accurate, both in an absolute and relative sense. The swarm is globally tied down by the GNSS-aided member and will rotate around it as the members' INSs drift while keeping relative positions intact. It is worth noting that while the relative position error significantly improves, the individual range errors do not change.
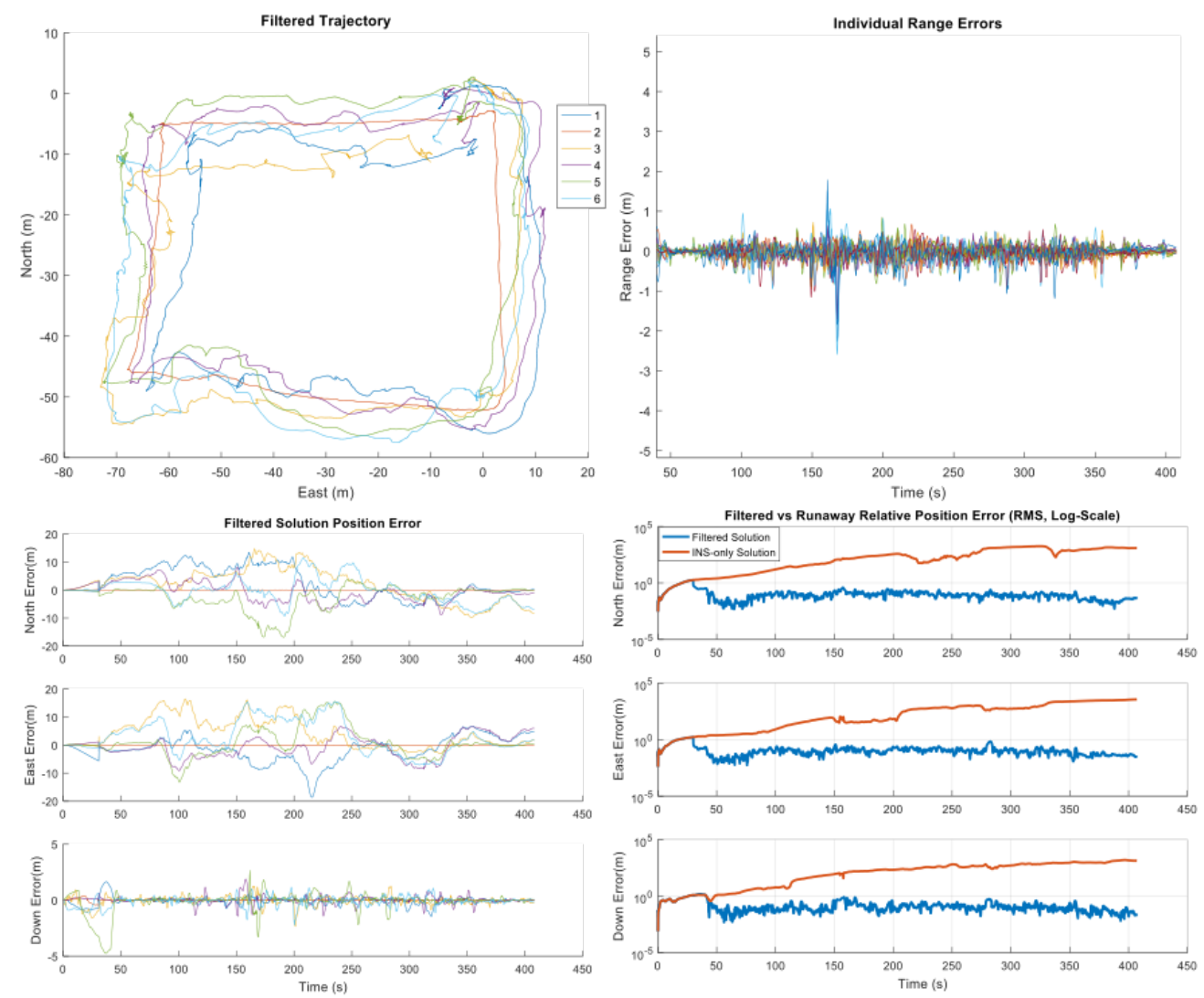

Figure 11. Filtered results with one GNSS-enabled member.

\subsection{Two GNSS-Enabled Members}

Next, a second member was equipped with a resilient GNSS receiver. Operating with two points of constraint as well as having the z-axis constraint by the baro-altimeters, the individual solutions improve dramatically as can be observed in Figure 12. When the filter settles after about 30 seconds, the solution with two GNSS-aided members (Octocopters 2 and 6) becomes even more accurate than with one GNSS-enabled member - the absolute error drops to near-zero from a $\pm 20 \mathrm{~m}$ fluctuation in the one-GNSS-enabled configuration. At this point, all three dimensions are tied down $-x$ and $y$ from the two GNSS-aided members, and $z$ from the baro-altimeter. One can observed 
that this solution occasionally goes into error, but then quickly returns. This can most likely be attributed to short periods of increased range error.
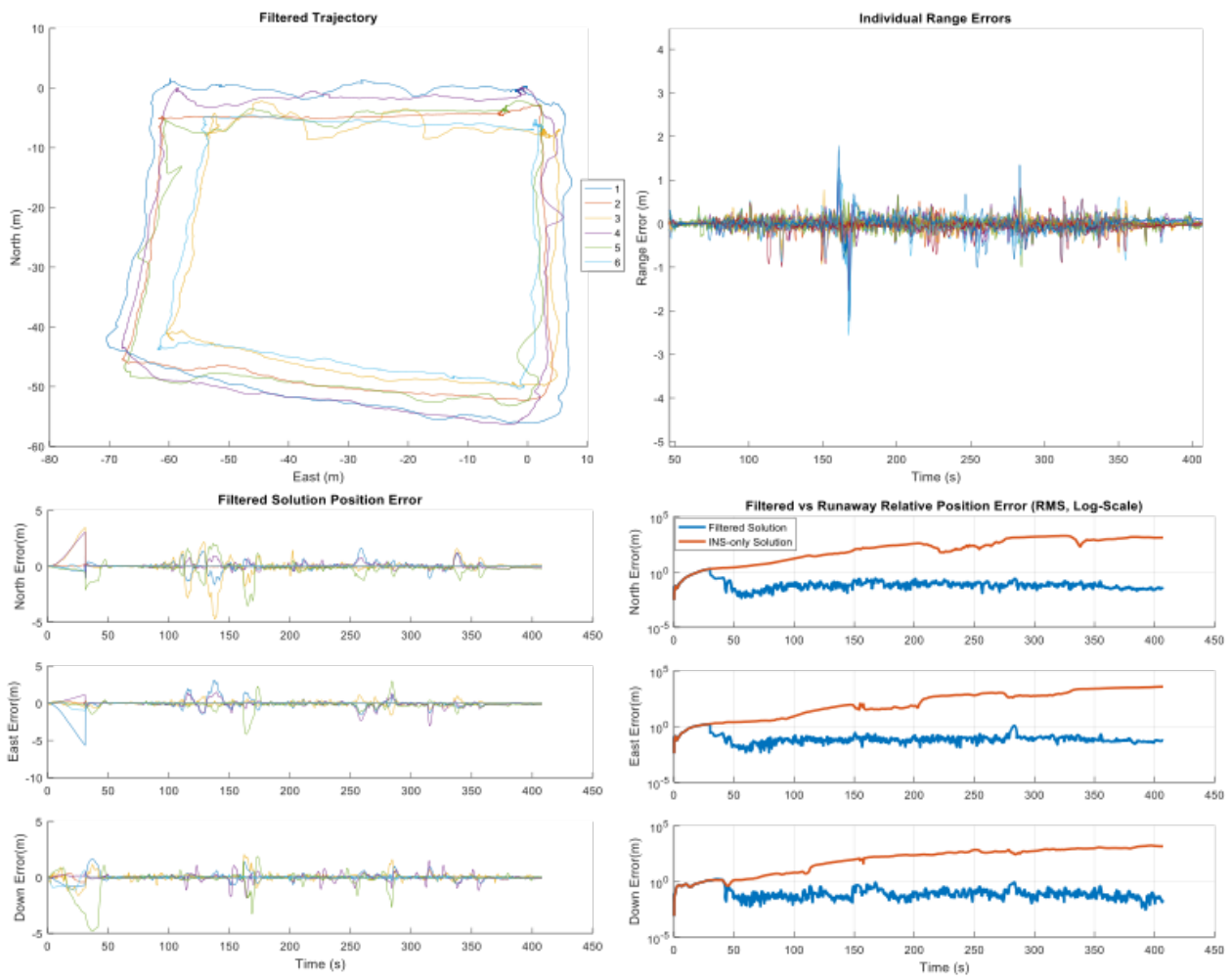

Figure 12. Filtered results with two GNSS-enabled members.

\subsection{Three GNSS-Enabled Members}

Finally, a third GNSS-enabled member was added (so, 50\% of the swarm has GNSS, and 50\% hasn't). With three points of constraint, the solution should improve, but diminishing returns are expected as the solution becomes is over-constrained. The results of this test are shown in Figure 13. As expected, adding the third GNSS-aided member (now 2, 5, and 6) creates yet a better solution. Most ambiguities are now solved, and more certainty is being established in the filter. The relative position error is consistently low and any errors in a member's position get corrected almost immediately. Even the individual range errors show signs of improvement. 

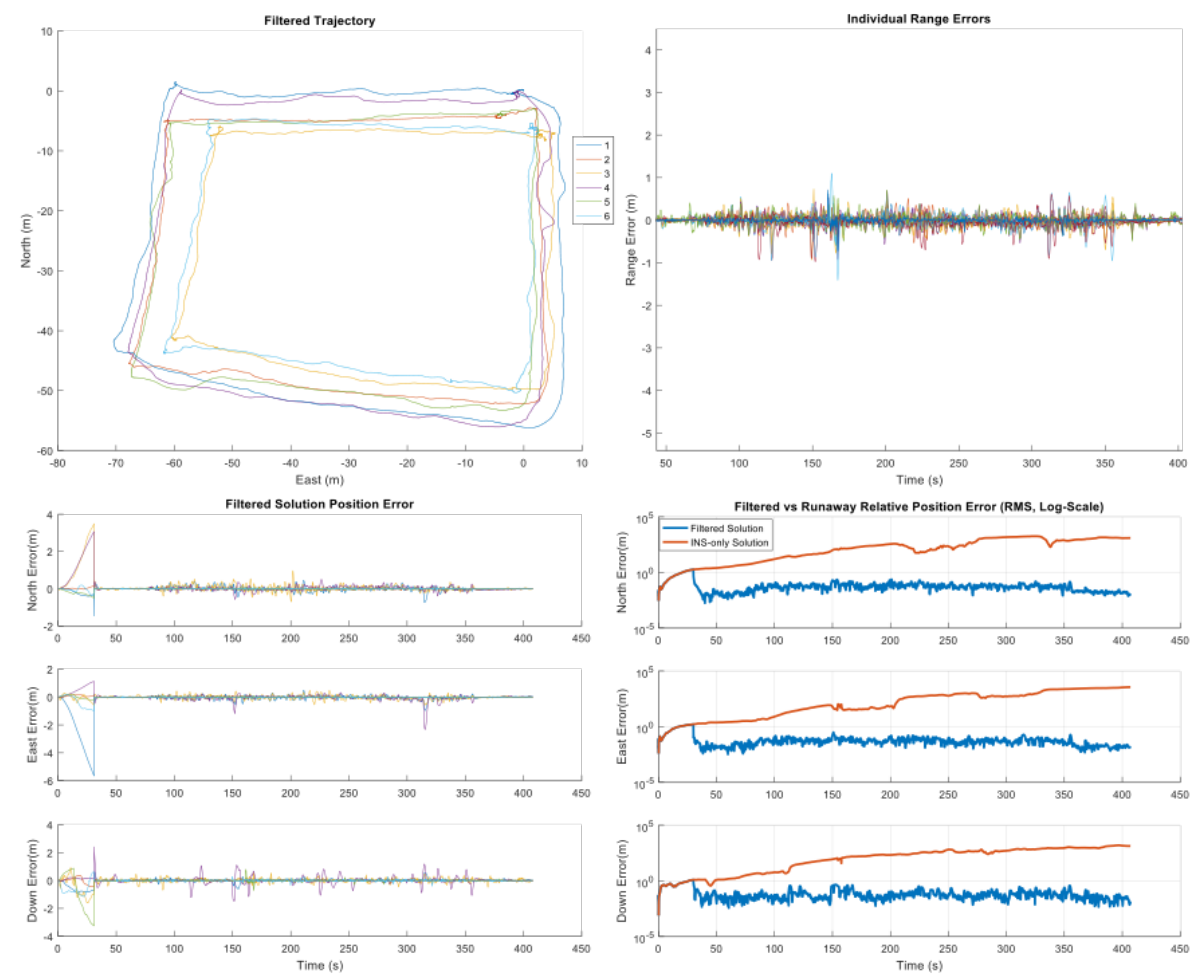

Figure 13. Filtered results with three GNSS-enabled members

\subsection{Filter Results when Flying through an Outage Zone}

In addition to evaluating the absolute and relative performance in the presence of zero or more GNS-enabled members, tests were performed to assess how well the swarm can navigate through a local GNSS-denied area. Figure 14 shows an example of the sUAS flight trajectories with an overlaid outage zone, where GNSS is not available

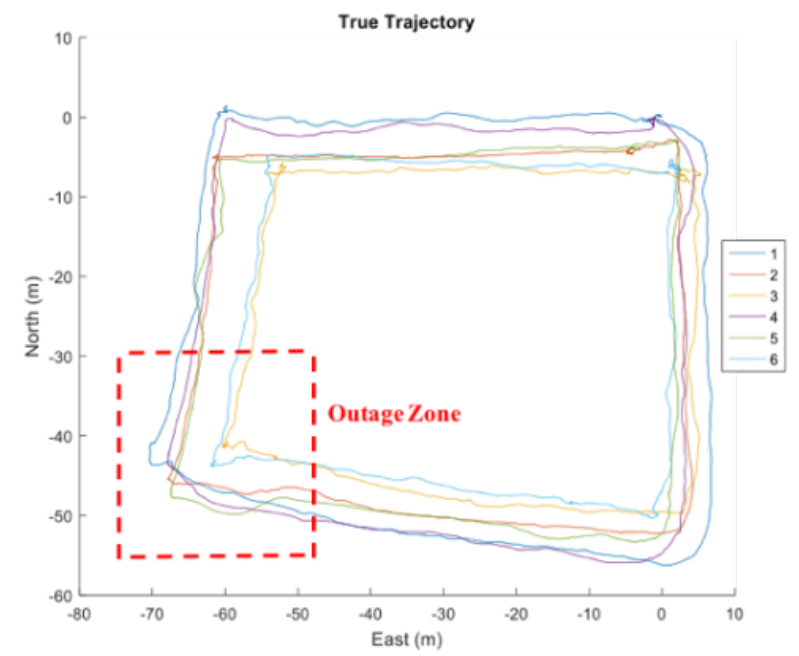

Figure 14. GNSS outage zone. 
Using just a loosely-coupled GNSS-Inertial filter, the results are poor when the swarm moves through the outage zone, as the absolute position is just coasting on the individual INS solutions.

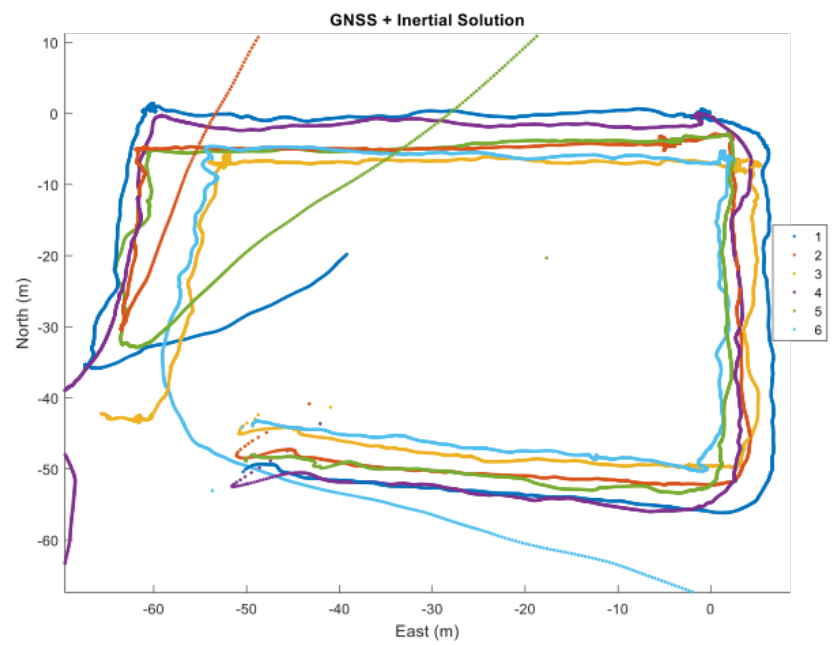

Figure 15. GNSS-Inertial solution.

Using the proposed method instead, the absolute position drift during the outage is reduced as illustrated in Figure 16.

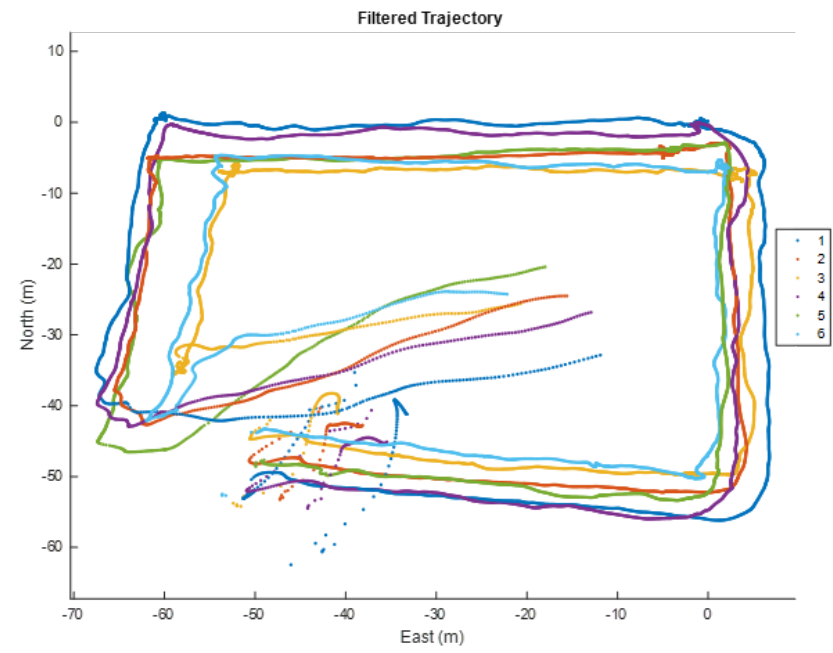

Figure 16. Filtered solution through GNSS outage zone.

As expected, the filtered solution performs better than the GNSS/INS position solution. There is still a significant amount of error, but the swarm is constrained to drift together (i.e., a low relative position error) and there is less discontinuity when the members exit the outage zone. During the outage, the peak individual error is around $2 \mathrm{~m}$ for the filtered solution, while the GNSS/INS reaches over $150 \mathrm{~m}$.

The next test assumes that one member of the swarm has technology that allows it to operate in the GNSS-denied zone. In a real-world scenario, this could represent one member having an 
advanced anti-jam/anti-spoof GNSS receiver, or a visual odometry system with an absolute position capability. The results are shown in Figure 17.

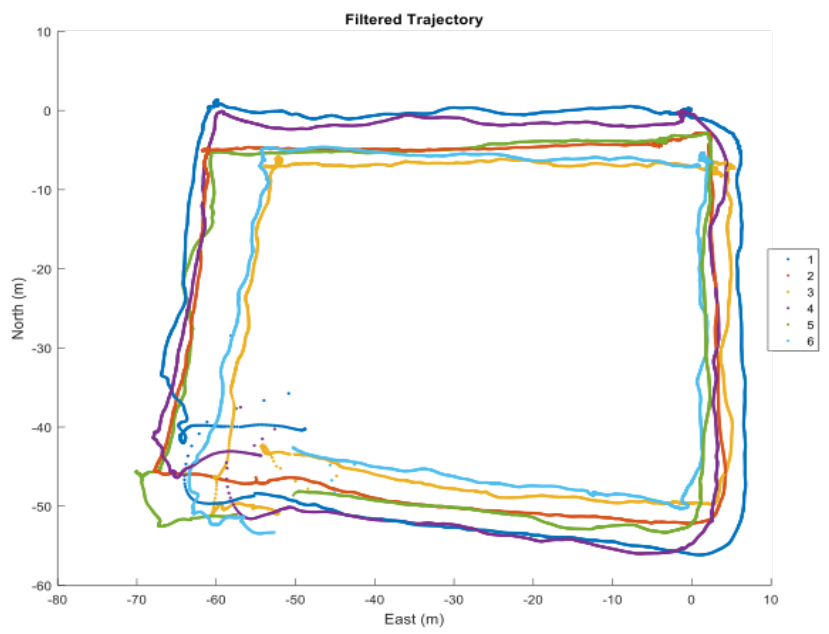

Figure 17. GNSS outage with one resilient member.

With one member equipped with more advanced technology, the entire swarm benefits when moving through the outage zone with less drift. Unfortunately, the swarm is not completely constraint in the absolute coordinate frame: it is still free to slowly rotate about the resilient member. This causes small discontinuities, though they are much smaller than those observed in Figure 16.

Adding one more resilient member should create a near-perfect navigation over the small outage zone as shown in Figure 18.

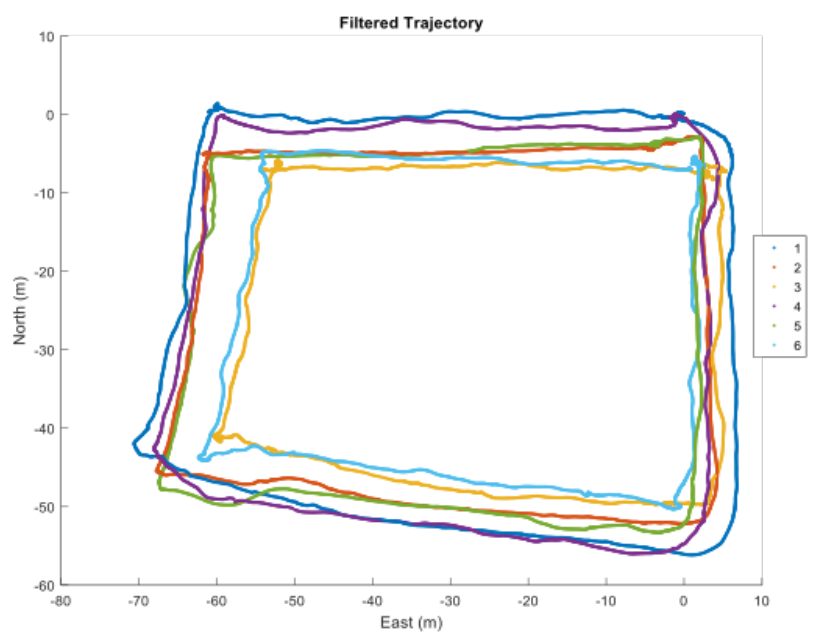

Figure 18. GNSS outage with two resilient members. 


\subsection{Flying Through Outage with Ground Speed Sensor}

If all members of the swarm loose GNSS capability during the outage section, it should be possible to navigate (though not as well) based off types of sensors. In the following example, a number of members are simulated as equipped with a ground velocity sensor (e.g., 2D optical flow) during the outage zone. This ground velocity sensor is simulated with a random walking bias, starting in the range of $\pm 0.2 \mathrm{~m} / \mathrm{s}$, and gaussian noise with a standard deviation of $0.1 \mathrm{~m} / \mathrm{s}$. The ground speed sensor can then be combined with an estimate of the sUAS attitude to obtain a velocity estimate, which is then fed into the filter. The outage zone is the same corner outage as in Figure 14.

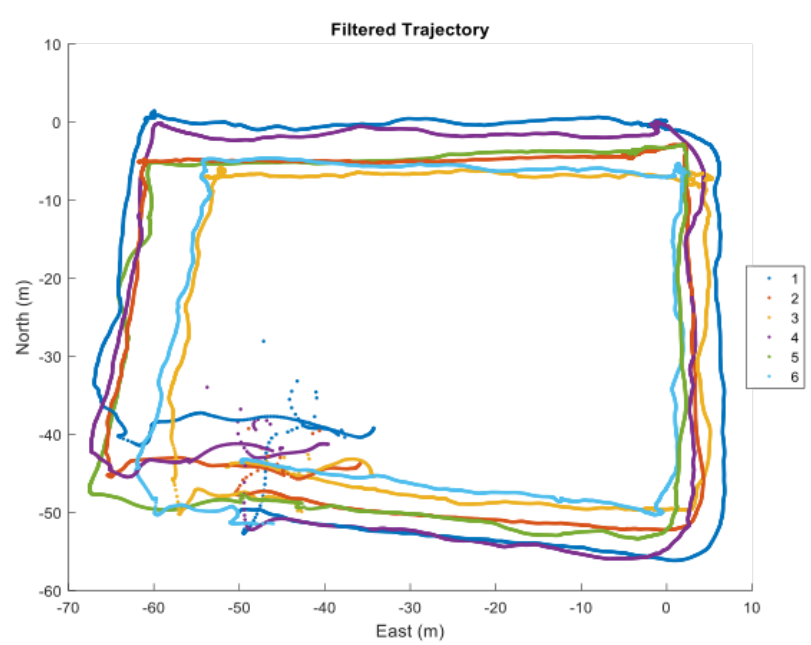

Figure 19. Corner outage zone with one ground-speed enabled member.

From Figure 19 it can be seen that the swarm (with one member equipped with a ground-velocity sensor) does not navigate as well as with a single GNSS-enabled member, but, for being completely GNSS-denied, the swarm functions well - it does not drift too far, and the relative position error remains low. This shows the filter's capability to utilize and distribute different types of sensor measurements. When adding any additional velocity sensor, further improves the results as shown in Figure 20.

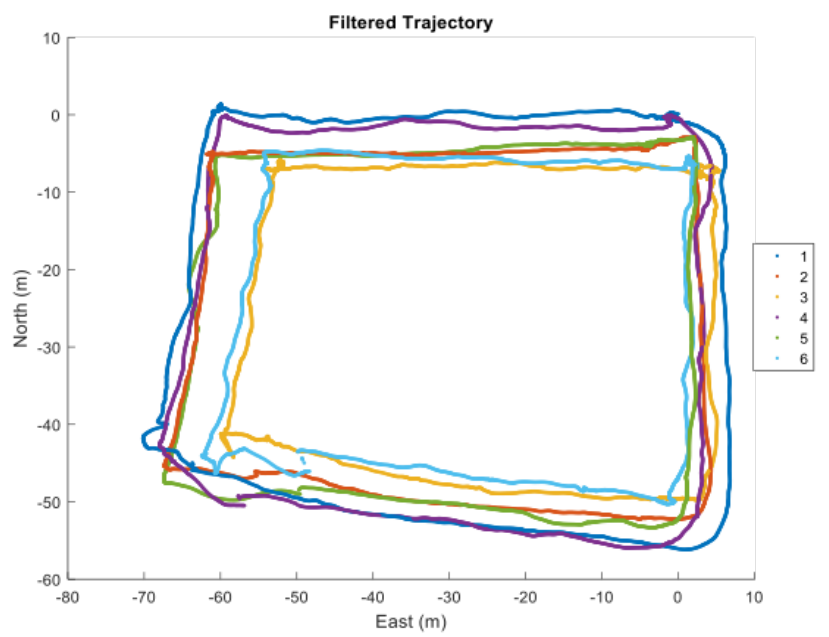

Figure 20. Corner outage zone with two ground-speed enabled members. 


\section{SUMMARY, CONCLUSIONS AND FUTURE WORK}

This paper presents a method that performs a centralized integration of inertial, UWB and barometric altimeter measurements, to enable relative and absolute navigation during GNSS outages. The method successfully constrained the inertial navigation solution with the new range radio measurements during these outages. During times when GNSS was available to one or more members, the filter was also capable of using these members to tie down the swarm to the navigation frame, effectively increasing the accuracy of every member of the swarm. This filter performed well in short GNSS outages, even with no GNSS-resilient members. This is useful in an urban canyon or other GNSS-denied environments, when performing a cooperative sUAS task. With only small modifications, the filter can be adapted to accept other types of sensor readings such as absolute position (GNSS) or ground velocity sensors.

Future work is needed to expand, analyze, and optimize this system. Many sensors exist that can provide navigation aid to an individual member, and therefore the entire swarm. Furthermore, decentralized version of the method should be addressed and versions that only use partial information to reduce the burden on the communication network. Also, a flight using many (6+) sUAS is planned, with actual range radio sensors and the proposed filter running in real-time.

\section{ACKNOWLEDGEMENT}

All flight tests used to evaluate the results here were funded by the Ohio University Russ Legacy Development Fund. The authors also want to thank Russell Gilabert and Adam Schultz for their help during the flight tests.

\section{REFERENCES}

[1] J. S. Álvares, D. B. Costa and R. R. Melo. Exploratory study of using unmanned aerial system imagery for construction site 3D mapping.

[2] S. Puliti, H. O. Ørka, T. Gobakken and E. Næsset, "Inventory of Small Forest Areas Using an Unmanned Aerial System," Remote Sens, vol. 7, no. 8, pp. 9632-9654, 2015.

[3] J. Capitan, L. Merino and A. Ollero, "Decentralized Cooperation of Multiple UAS for Multi-target," in International Conference on Unmanned Aircraft Systems, Orlando, FL, USA, 2014.

[4] A. Schultz, R. Gilabert, B. Akshay, M. Uijt de Haag and Z. Zhu, "A navigation and mapping method for UAS during under-the-canopy forest operations," in Proceedings of the IEEE/ION Position, Location and Navigation Symposium, Savannah, GA, USA, 2016.

[5] J. E. Huff and M. Uijt de Haag, "Assured relative and absolute navigation of a swarm of small UAS," in Proceedings of the IEEE/AIAA 36th Digital Avionics Systems Conference (DASC), St. Petersburg, FL, USA, 2017.

[6] J. Huff, Absolute and Relative Navigation of an sUAS Swarm Using Integrated GNSS, Inertial and Range Radios, M.S.E.E. Thesis, Ohio University, 2018.

[7] J. Hol, Sensor Fusion and Calibration of Inertial Sensors, Vision, Ultra-Wideband and GPS, Linkoping: Linkoping University, 2011. 
[8] M. Kok, J. D. Hol and T. B. Schon, Indoor positioning using ultrawideband and inertial measurements, 2015.

[9] A. Gelb, Applied Optimal Estimation, MIT Press, 1974.

[10] J. L. Farrell, GNSS Aided Navigation \& Tracking - Inertially Augmented or Autonomous, America Literary Press, 2007.

[11] P. D. Groves, Principles of GNSS, Inertial, and Multisensor Integrated Navigation Systems, Artech House, 2008.

[12] B. Southall, B. F. Buxton and J. A. Marchant, Controllability and Observability: Tools for Kalman Filter Design, Wrest Park, Silsoe, Bedfordshire: Silsoe Research Insitute.

[13] B. K. Horn, H. M. Hilden and S. Negahdaripour, Closed-Form Solution of Absolute Orientation.

[14] "http://www.ros.org/," [Online]. [Accessed July 2018]. 